\title{
Experimental Work With Photonic Band Gap Fiber: Building A Laser Electron Accelerator
}

\author{
Melissa Lincoln, Rasmus Ischebeck, Robert Noble, Robert Siemann \\ Advanced Accelerator Research Department, Stanford Linear Accelerator Center, 2575 Sand Hill \\ Road, Menlo Park, CA 94025, USA
}

\begin{abstract}
In the laser acceleration project E-163 at the Stanford Linear Accelerator Center, work is being done toward building a traveling wave accelerator that uses as its accelerating structure a length of photonic band gap fiber. The small scale of the optical fiber allows radiation at optical wavelengths to be used to provide the necessary accelerating energy. Optical wavelength driving energy in a small structure yields higher accelerating fields. The existence of a speed-oflight accelerating mode in a photonic band gap fiber has been calculated previously [1]. This paper presents an overview of several of the experimental challenges posed in the development of the proposed photonic band gap fiber accelerator system.
\end{abstract}

Keywords: Photonic band gap fiber.

PACS: 41.75.Jv, 42.79.Gn, 42.81.-i, 41.75.Ht.

\section{INTRODUCTION}

The construction of a fiber-based accelerator requires thorough characterization of the candidate fiber structure. It is crucial to the successful operation of the accelerator that the frequency of the driving laser light be selected so as to fall within a band gap of the fiber. Thus, the transmission spectrum of the fiber must be well known. The fiber's band gap describes a set of electromagnetic modes that are disallowed from propagating transversely, and thus may only travel longitudinally through the fiber. Radiation within the band gap cannot be lost radially through the fiber, then, for the fiber acts as a radial reflector [2]. A requirement for acceleration is that the chosen frequency can excite a mode with speed of light phase velocity. In order to be able to excite an accelerating mode in the fiber, it must also be well-known how to properly couple light into the fiber structure to achieve desired modes.

Once an understanding of the candidate photonic structure is developed, an effort must be made to control certain parameters in the specific piece of fiber used as accelerating structure. It is desirable to minimize loss and back-reflection when coupling the laser and electron beams into the fiber structure, so techniques to create an optically smooth surface on each end face of the fiber must be investigated. Due to dispersive effects within the fiber, the laser's pulse shape will experience slippage with respect to the relativistic electron beam. For a laser pulse duration of picoseconds, the fiber structure's length must be restricted to on the order of $1 \mathrm{~mm}$ [3]. The laser pulse must also have the appropriate phase velocity to allow it to optimally 
copropagate with the electron bunch. It is well known that the phase velocity of radiation through a dielectric structure is sensitive to such environmental factors as temperature. Changes in temperature will change the fiber's diameter and refractive index, which will change the phase velocity of the laser beam within it. This indicates the necessity to control the fiber's temperature as well. Another important characteristic to control is the polarization of the incident laser beam. Only radially polarized light will generate a completely longitudinal electric field within the fiber structure [4], so an optical system must be developed to reliably generate it.

\section{WORK IN PROGRESS}

Surmounting the aforementioned experimental challenges has required the investigation of a varied set of methodologies.

\section{Fiber Cleaving}

First, the generation of smooth faces on the fiber is achieved by proper cutting, or cleaving, of the fiber. A cleave in an optical fiber is a delicate, controlled break which is intended to create an optically flat end face, perpendicular to the longitudinal axis of the fiber. A cleave is made by first introducing a small fracture, or nick, into the fiber using a sharp blade of some hard material, such as diamond. Proper tension is then applied to the fiber and the fracture will propagate in a controlled fashion, creating the desired end face [5]. See Figure 1.

Cleaving can be performed using an electronic or manual machine that creates the nick and applies tension

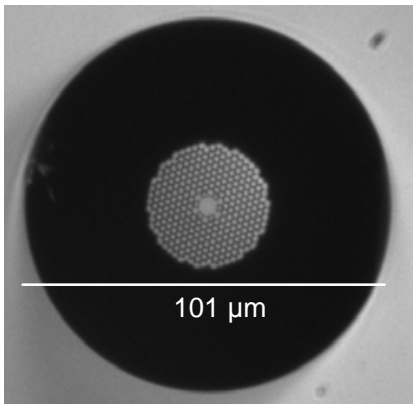

FIGURE 1. Photograph of cross-section of photonic band gap fiber used in this work. automatically. Cleaving can also be carried out by hand by a skilled operator; it has been determined that for the purposes of the current project, hand-cleaving is the preferred technique. Hand-cleaving allows great flexibility in the choice of fiber diameters. Current commercially available mechanical and electronic cleavers have a set diameter of fiber which they are designed to cleave, and making changes to this setting is either impossible or expensive and time-consuming. Hand-cleaving poses no such restrictions; nearly any arbitrary diameter of fiber can be cleaved by hand using the same set of tools. It will be necessary in this project to work with at least two different diameters of fiber-one designed for visible-wavelength operation, which is used for characterization studies, and another larger one (a scaled-up version) designed for infrared operation, which will be used as the structure in the final accelerator-so the consideration of diameter flexibility is nontrivial.

However, cleaving fiber by hand introduces difficulty in controlling the length of the fiber accurately. Length control is limited by the steadiness of the cleaver's hand and the resolution of the cleaver's vision. Clearly, length error estimates for this technique are variable and difficult to reliably establish. Yet, this fault in handcleaving may be outweighed by the fact that it also provides the advantage of greater 
choice in cleaved fiber length. Current commercially available mechanical and electronic cleavers have set minimum cleaved fiber lengths, usually on the order of several centimeters. Machines are simply not designed to cut pieces of fiber on the millimeter scale, as the mainstream fiber optic industry does not frequently require such short fiber pieces. A person cleaving fiber by hand can make use of fine manipulation instruments such as tweezers to achieve millimeter-length fiber pieces. As previously mentioned, fiber pieces of no more than about a millimeter's length are crucial to this project.

\section{Radially Polarized Laser Mode And Coupling To Fiber Structures}

Once surfaces are created on the ends of the fiber that will allow coupling to a laser beam, it is natural to look at the issue of actually creating the type of laser light needed. The accelerating mode can be created by radially polarized light [6]. An optical system for the reliable generation of radially polarized light has been fabricated. Its operation centers around splitting a Gaussian beam, dephasing one part of each wave front, linearly polarizing the wave fronts, and then interferometrically recombining them.

The next item to address is how to couple said laser into the fiber structure. Efficient coupling of laser light into a fiber structure requires that the laser be within the numerical aperture of the fiber. This is achieved via the use of a microscope objective to focus the laser beam onto the cleaved end face of an extended piece (longer than 1 millimeter) of the fiber. The fiber's position is then adjusted iteratively to achieve the desired mode. The output cleaved end face of the fiber is observed with a camera, and the input end is placed on a three-dimensional micrometer stage in front of the microscope objective. The input end's position is then adjusted until the camera sees the desired near-field output mode profile.

For characterization studies, the experimental setup is simple. However, for the prototype laser accelerator, input coupling will be slightly more complex. In order to couple both the electron beam and laser into the fiber, the laser beam will need to be brought in from off-axis through the use of a pellicle. The output mode profile must also be observed in the same manner. Clearly, the prototype accelerator scheme will require microscope objectives of long working distances of several centimeters.

\section{Fiber Mode Characterization And Numerical Simulation}

The next question to ask is how to characterize the output modes observed. The output near-field intensity profiles recorded with the camera observing the output end of the fiber are compared to mode profiles generated via numerical simulation. Current work involves several different candidate fiber modeling codes, such as the Center for Ultrahigh-Bandwidth Devices for Optical Systems's Microstructured Optical Fibres Utilities (CUDOS) [7], RSoft Design Group's BandSOLVE [8], and MIT Photonic Bands (MPB) [9]. To determine which code is most appropriate for the purposes of this experiment, and which parameters are most important to model, simulation must be compared with real observations. The near-field intensity profiles 
of the fiber's output light are taken for a variety of modes and are compared with the predictions of numerical modeling codes.

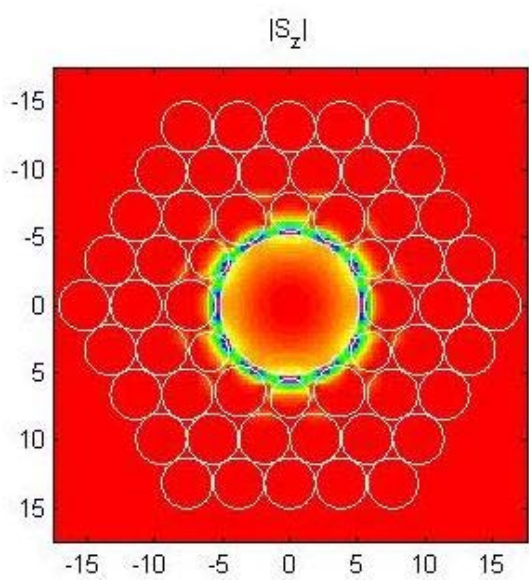

FIGURE 2. Field plot of an accelerating mode simulation of Poynting vector $\mathrm{S}_{\mathrm{z}}$ created by CUDOS.
$\left|E_{z}\right|$
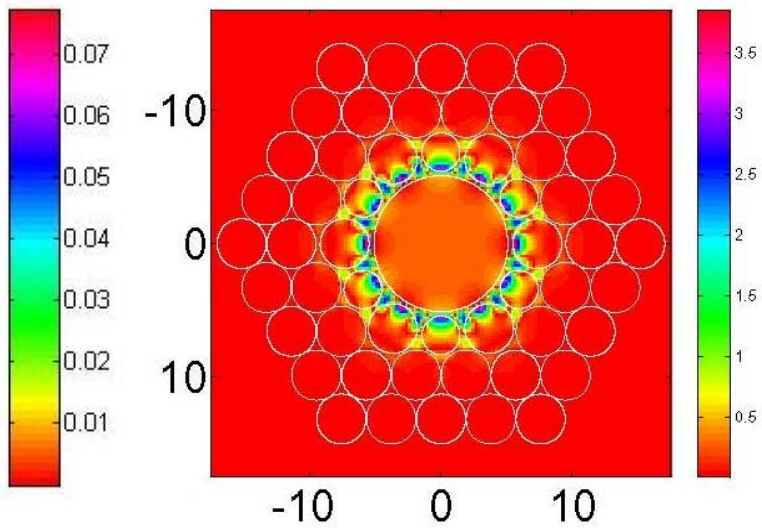

FIGURE 3. Field plot of an accelerating mode simulation of longitudinal electric field $\mathrm{E}_{\mathrm{z}}$ created by CUDOS.

The modes studied do not necessarily have to be accelerating modes in order to be revealing; for example, the fiber's telecom design mode could be used as one metric for numerical simulation performance. A good theoretical model is needed for this experiment, so non-accelerating modes are important in their own right. A proper theoretical model will guide the selection of the appropriate laser wavelength at which to run an optical parametric amplifier that will provide the optical driving energy for the experiment.

For the sake of illustration, a pair of plots produced by one of the numerical modeling programs, CUDOS, are shown in Figures 2 and 3. They represent the magnitude of the Poynting vector and the longitudinal electric field and for a simulated accelerating mode. The Poynting vector plot corresponds to the intensity profile that should be observed for this mode. Note that within the large central defect of the fiber there is an essentially constant, electric field, which should be expected for an accelerating mode. It is clear that there is a good deal of electric field around the edge of the central defect that would not be of use in accelerating an electron beam and could damage the structure. So this mode is non-ideal. However, simulations with the CUDOS code (and others) are still a work in progress for this project, and there exist many accelerating modes. The mode that will be finally selected for acceleration will have good Poynting flux and $\mathrm{E}_{\mathrm{z}}$ concentrations.

\section{Transmission Spectrum of Fiber}

It was already stated that the correct numerical model will lead to the proper wavelength of operation. The wavelength of laser light that is passed through the fiber must be calculated so as to lie in the fiber's band gap. It is important to verify that the fiber's actual band gap does indeed correspond well to calculation, so an experimental 
measure of the fiber's band gap should be taken. This is achieved via measurement of the transmission spectrum of the fiber. The wavelengths that are guided through the fiber and appear at the fiber's output must lie within the fiber's band gap. The experimental setup for this measurement is simple to characterize. White light is passed through the fiber structure so as to be observable by a fiber-coupled spectrometer. In Figure 4 is seen a black-and-white photograph of a particular white light core mode transmitted through the fiber (its design wavelength is $633 \mathrm{~nm}$ ), and in

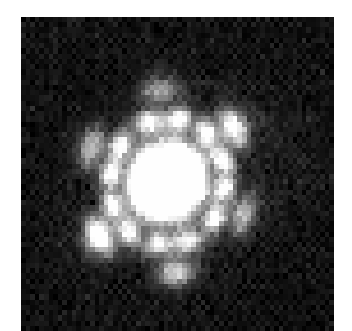

FIGURE 4. Near-field photograph of white light core mode propagated through fiber pictured in Figure 1.

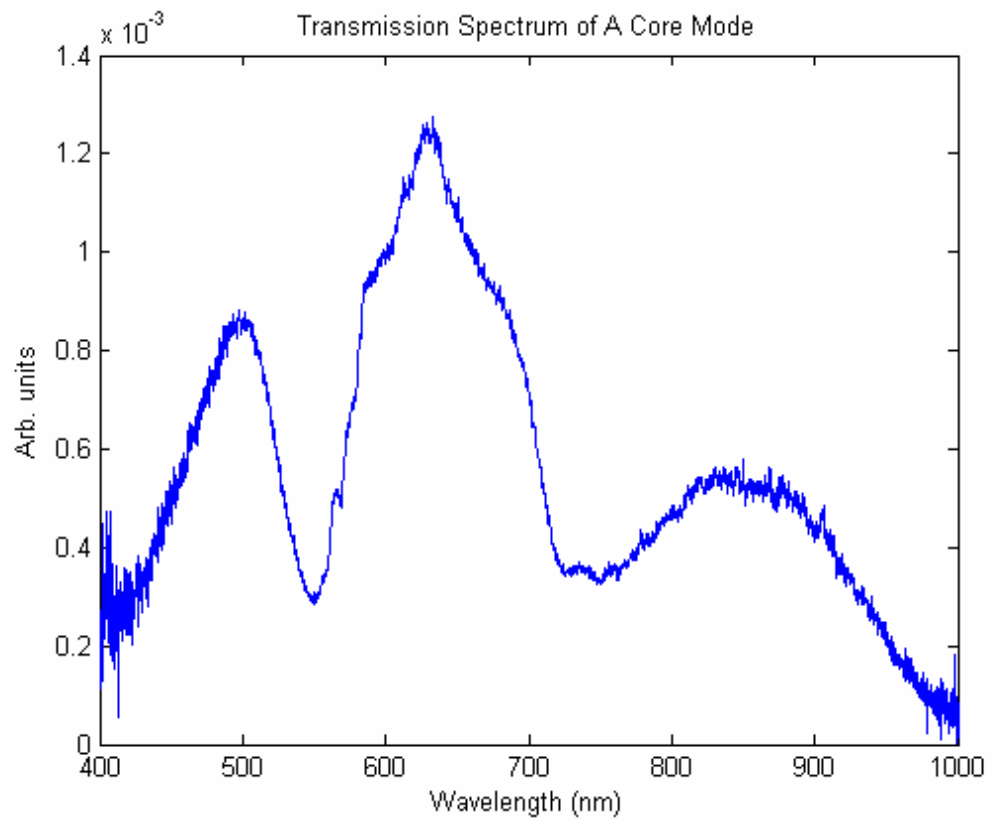

FIGURE 5. Transmission spectrum measured across the visible wavelength range for the white light core mode shown in Figure 4. Note that this is not a percentage transmission plot; the $y$-axis units are arbitrary.

Figure 5 is seen the resulting transmission spectrum measured for this mode. Different core mode patterns have different transmission spectra. Note that the mode shown in Figure 4 is not an accelerating mode; it has been illuminated with incoherent white light of random polarization.

It is clear to see that this mode has several spectral peaks; one is expected and seen at $633 \mathrm{~nm}$, the design wavelength. Other spectral peaks are seen at approximately $500 \mathrm{~nm}, 580 \mathrm{~nm}, 680 \mathrm{~nm}, 830 \mathrm{~nm}$, and $880 \mathrm{~nm}$. Current work consists of characterizing mode patterns and their spectra in such a way as to determine the spatial discrimination in the transport of different frequencies of light. This is achieved by frequency filtering the white light input to the fiber structure, photographing the resulting output core mode, and seeking out correlation between frequency and mode shape.

\section{PLANNED WORK}

An important planned measurement is of the stability of the phase velocity of the laser light that passes through the fiber under drifts in the fiber's temperature. The 
silica that makes up the fiber will expand with increasing temperature, and its index of refraction will change. The fiber will lengthen, and the phase velocity of the light being guided through the fiber will be affected. It is important to characterize this effect so that it can be properly mitigated in the final experimental setup. A Michelson-type interferometer as shown in Figure 6 will be built. Instead of having a mirror on an expandable arm, the free-space laser will be coupled into a length of fiber in one arm. This fiber will be placed in contact with a heater, and the change in phase induced by varying the fiber's temperature will be measured. Thus, it will be determined how tightly the fiber's temperature in the accelerator will need to be controlled. This Michelson setup can also be used to test the phase velocity's tolerance of other environmental conditions, such as pressure and vibration applied to the fiber.

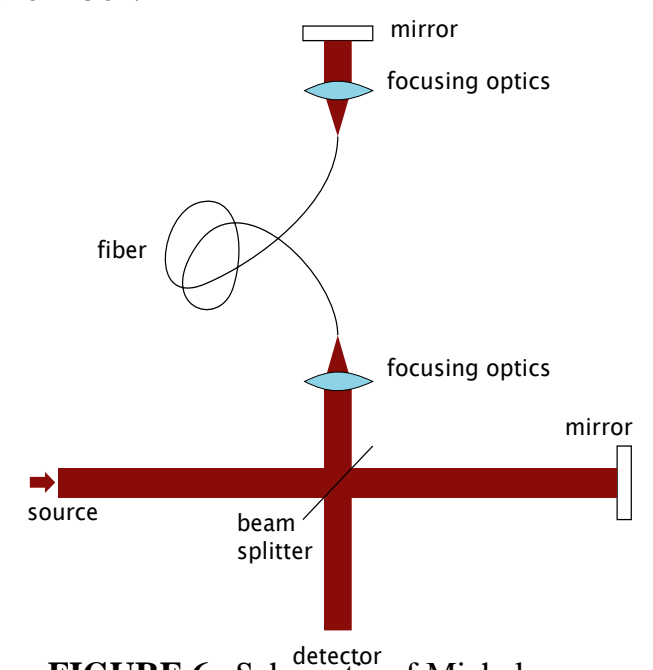

FIGURE 6. Schematic of Michelson Interferometer to be used to study fiber's tolerance of varying environmental conditions.

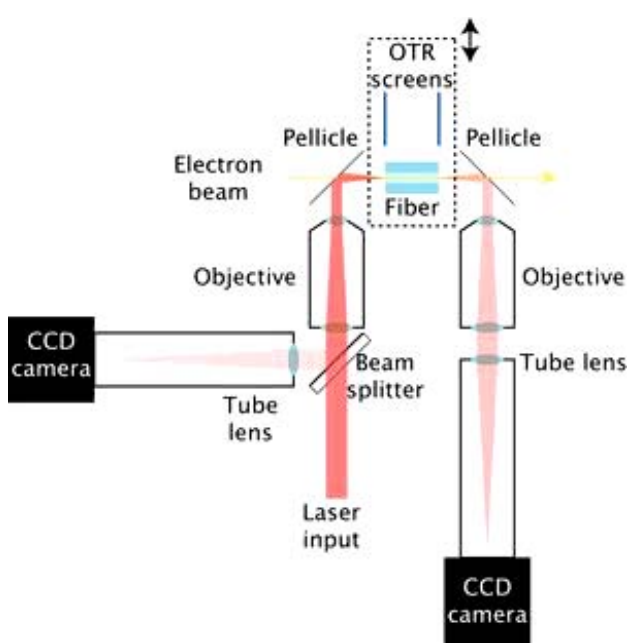

FIGURE 7. Schematic of candidate setup for proof-of-principle acceleration of electrons through fiber structure using laser driving energy.

The actual details of the accelerator's setup also need to be finalized. A method to best couple into the fiber both the electron beam and the laser light needs to be determined. One candidate scheme for achieving this is shown in Figure 7. The laser mode can be observed both entering and exiting the fiber structure by two CCD cameras. Pellicles on either side of the fiber allow the laser light to be brought in and then picked off without interruption of the electron beam. Other diagnostics can also be inserted into the beam line in place of the fiber in order to characterize the electron beam. Prototypes will need to be built and investigated as candidate accelerator schemes are developed. And of course, the final goal for future work is to successfully accelerate electrons in a proof-of-principle experiment that demonstrates the feasibility of photonic band gap fibers as accelerating structures.

\section{ACKNOWLEDGEMENTS}

Many thanks to members of the Advanced Accelerator Research Division at the Stanford Linear Accelerator Center - Eric Colby, Benjamin Cowan, Christopher 
Sears, James Spencer, and Dieter Walz. Their guidance and assistance in the fabrication of electronics and optical systems for this work is indispensable. Thanks as well to several researchers in the Applied Physics Department at Stanford University - Robert Byer, Michel Digonnet, Patrick Lu, Tomas Plettner, and Supriyo Sinha. Their assistance and the lending of their expertise in working with fiber optics are greatly appreciated. Work supported by US Department of Energy contracts DEAC02-76SF00515 and DE-FG03-97ER41043-II.

\section{REFERENCES}

1. Lin, Xintian Eddie. "Photonic band gap fiber accelerator." Phys. Rev. Special Topics - Acc. And Beams 4.051301 (2001): 1-7.

2. Bjarklev, Anders, Jes Broeng, and Araceli Sanchez Bjarklev. Photonic Crystal Fibres. Kluwer Academic Publishers, Boston, 2003, p. 20.

3. Lin, 3 .

4. Lin, 5.

5. Hobbs, Philip. Building Electro-Optical Systems: Making It All Work. Wiley \& Sons, New York, 2000, p. 263.

6. Lin, 5.

7. White, T., et al. "Multipole method for microstructured optical fibers I : formulation.” J. Opt. Soc. B. 19, pp. 2322-2330 (2002).

8. http://www.rsoftdesign.com/, accessed August 28, 2006.

9. Johnson, Steven and J. D. Joannopoulos. "Block-iterative frequency-domain methods for Maxwell's equations in a planewave basis." Optics Express 8. 3, 173-190 (2001). 